\title{
AUGMENTATION OF AN INSTITUTE'S KITCHEN: AN AMBIENT AUDITORY DISPLAY OF ELECTRIC POWER CONSUMPTION
}

\author{
Katharina Groß-Vogt ${ }^{1}$, Marian Weger ${ }^{1}$, Robert Höldrich ${ }^{1}$, \\ Thomas Hermann ${ }^{2}$, Till Bovermann ${ }^{3}$, Stefan Reichmann ${ }^{4}$ \\ ${ }^{1}$ IEM - Institute of Electronic Music and Acoustics, \\ University of Music and Performing Arts Graz, Austria, \\ ${ }^{2}$ Ambient Intelligence Group, CITEC, Bielefeld University, Germany, \\ ${ }^{3}$ University of Applied Arts Vienna, Austria, \\ ${ }^{4}$ Center for Social Research, University of Graz, Austria. \\ vogteiem. at
}

\begin{abstract}
Efficient feedback on energy consumption is regarded as one step towards a more sustainable lifestyle. Sonification is very apt to convey such information continuously in an ambient and effective way. This paper presents a pilot system for sonifying the electric power consumption of an institute's kitchen. The reverberation of the kitchen is changed depending on the actual consumption and its difference to a weekly baseline. If the actual consumption is low, it is mapped to a plausible kitchen reverberation. If it is high compared to the baseline, the reverberation becomes unnatural. Evaluating the system gave insights on perceptibility and acceptance of auditory augmentation in a semi-home context.
\end{abstract}

\section{BACKGROUND}

Electricity consumption is a key issue in striving towards sustainability. Households in the EU are consuming $29 \%$ of final electrical energy, excluding energy used for transport [1]. Household devices are becoming more efficient, but at the same time are increasing in number and functionality. Therefore, the overall inhome electricity use is rising and solutions to a more careful use of electricity are needed. By 2020, smart meters (intelligent, digital electric meters) are predicted to be implemented in approximately $72 \%$ of European households [2]. In addition, consumer electronic devices are gaining more and more networking functionalities - by 2020, the Internet of Things (IoT) in the EU-28 is expected to number more than 6 billion devices [3]. This opens up to the possibility to access real-time information for home monitoring.

Numerous studies on smart meters in recent years have established the fact that feedback has measurable effects on behavior [4]. Typical forms of feedback are smart meters with in-home displays or web portals, informative billing, or similar [5]. Some current approaches visualize energy consumption metaphorically as flowing water [6], or even use a real indoor fountain for ambient sonification [7]. Schwartz et al. relate to energy as an imperceptible entity [8]. Therefore, design strategies have to make it perceptible in our everyday lives in order to allow for an emotional connection. Furthermore, the rationalistic paradigm is criticized in the design of standard systems for energy feedback - people often have difficulties apprehending the information provided by feedback systems because appropriate contextualization is missing.

The aim of our ongoing research is to take advantage of auditory display to support energy awareness in an ambient in-home setting. The pilot study presented in this paper is a first step towards a sonification system for in-home electrical power consumption (henceforth abbreviated as 'power'). As relevant factors for efficient feedback, both perceptibility and contextualization are being explored.

\section{AUGMENTED AUDIFICATION AND RELATED WORK}

The sonification of the pilot system described in this paper is based on changing the properties of the kitchen's acoustics by adding artificial reverberation. A large amount of literature deals with related approaches from environmental sociology, HCI, sonification, and sound and music computing. A short overview will be given in the following paragraphs.

The concept of auditory augmentation has been proposed by Bovermann et al. [9] as "building blocks supporting the design of data representation tools, which unobtrusively alter the auditory characteristics of structure born sounds". The concept has been discussed to be extended to the more general blended sonification, which "describes the process of manipulating physical interaction sounds or environmental sounds in such a way that the resulting sound carries additional information of interest while the formed auditory gestalt is still perceived as coherent auditory event." In the knock'knock blended sonification [10], reverberation of knocking the door was used to communicate whether somebody is present behind the office door. In this approach, the time span after the person has left the room is mapped to reverberation: an empty room sounds more reverberant than a stuffed one. The authors of knock'knock address the importance of a calm display. Auditory augmentation and blended sonification only work with active input, and are, therefore, calm in the sense of calm computing.

Kilander and Lönnquist presented the concept of WISP, or Weakly Intrusive Ambient Soundscape [11]. In a ubiquituous 


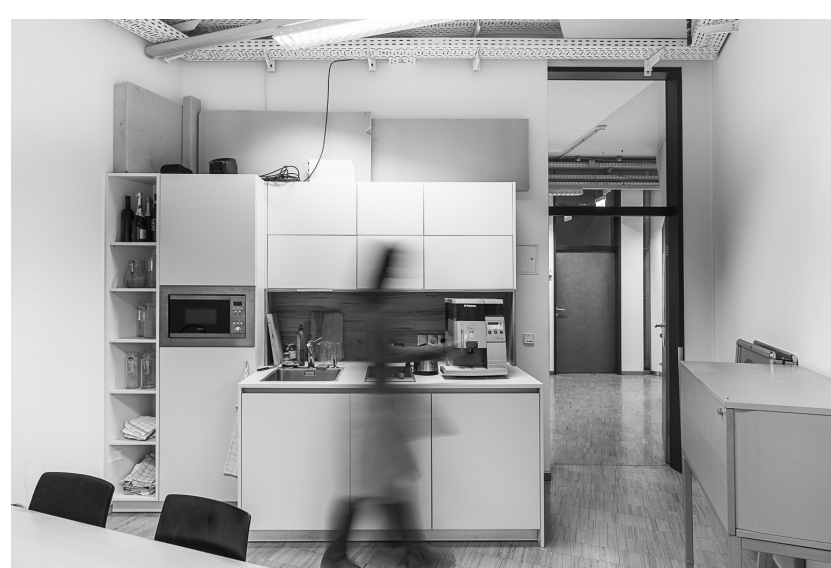

(a)

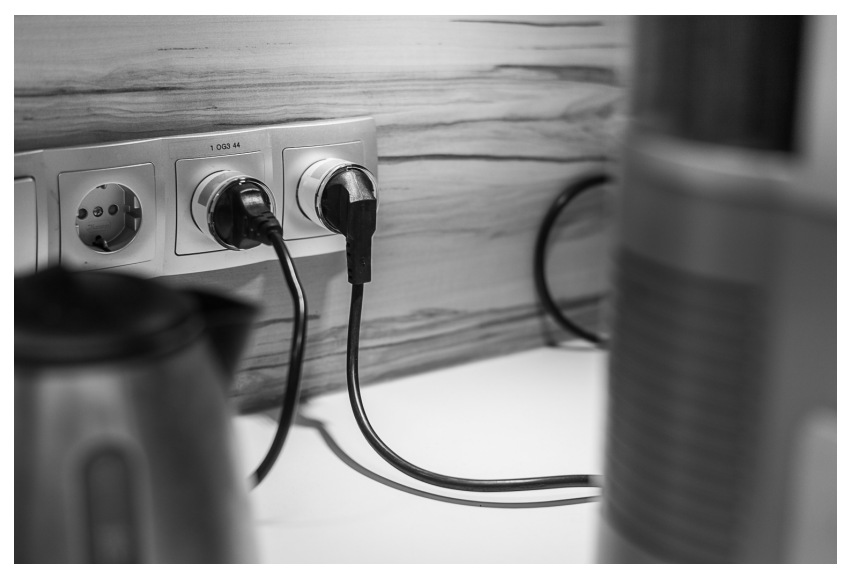

(b)

Figure 1: (a) Kitchen of the Institute of Electronic Music and Acoustics, IEM, Graz. (b) Wall plugs measuring and sending data on electric power consumption. Photos: Till Bovermann.

service environment, individual notifications have been presented with a sound associated to each user. Playback volume and reverberation have been used to convey three levels of intensity of the notification. High intensity was mapped to a dry, loud sound, while low intensity was saturated by reverberation, giving the impression of a far sound. The polarity of the mapping is opposite to our pilot study (see below) and it might be interesting to compare the two approaches to each other in a future study.

A similar project to WISP was realized by Brazil and Fernström [12] who explored a basic system for an ambient auditory display of a group. The presence of colleagues was sonified as soundscape of individual sounds, each time a person came or left the workplace. The proposed system has similarities to our pilot study in taking the work surrounding for a field test, but focusses on auditory icons for creating a soundscape instead of a calm approach.

Parthy et al. [13] also used reverberation for ambient data communication. They conducted a small study where they found that the reverberant decay time (RDT) has to increase by approximately $60 \%$ or decrease by approximately $30 \%$ in order to be clearly perceived. These findings are in approximate accordance with We- ber's law which states that perceived change in a stimulus is proportional to its size. Parthy et al.'s study was conducted with music as input signal and with adapted (identical) RMS amplitude for various RDT values. These factors might play a role in the way that the RDT has to be changed so drastically in order to be perceived. We assume in our approach that the sensitivity is higher when (1) the amplitude is used as additional parameter of reverberation and (2) the input signals are more transient than with musical input.

The works cited above reflect on the goals of ambient displays, e.g., awareness and lightweight interactions [12], to "convey an intuitive sense of any graspable process" rather than a direct information display [11], or a system that is "not intended to give a precise representation of the data value" [13]. Our pilot system follows the same idea, exploring perceptibility of un/-natural reverberation as a sonification parameter.

\section{THE AUGMENTED KITCHEN}

This paper presents a pilot study in a surrounding that facilitates both easy maintainance and constitutes a valid field test for an inhome setting: our institute's kitchen. An institute's kitchen is a specific surrounding, neither private nor really public. Furthermore, the users of the kitchen do not pay for the electric power consumption, as would be the case in an in-home setting. Therefore, the purpose of the study can only be to raise awareness of power consumption. No large behavioural changes are to be expected.

The kitchen is a 16-square-meters room equipped with a table for 8 people and a kitchen unit including
- (A) a dishwasher,
- (B) a coffee maker,
- (C) a water kettle,
- (D) a microwave oven and
- (E) a fridge.

The power of the above mentioned five appliances has been monitored and sonified, as discussed in the next sections. The kitchen unit also comprises an electrical stove, but this has been unplugged permanently for security reasons (as it has never been used and the water kettle is placed on it). Furthermore, electrical light was neglected, i.e. the last permanent appliance in the kitchen that drains power. Pictures of the kitchen and the wall plugs measuring the power are shown in Fig. 1. The kitchen is used regularly by 15 members of staff, all of whom have their workplace in the same building as the kitchen, and by external teachers, students and visitors of the institute. It is also used as a small conference room in case all other rooms are occupied.

Our sonification design is based on virtual acoustics. Acoustic properties of a room can be changed by adding reverberation. In the simplest case this means recording the environmental sound with a microphone, applying filters and playing it back over a loudspeaker in real-time. Care has to be taken to avoid electro-acoustic feedback loops. Such a systems of virtual acoustics is used frequently at our institute to showcase the power of audio to diverse audiences in our main performance and lecture hall, the CUBE [14]. Therefore, all our kitchen users, i.e. the test subjects for our pilot study, are familiar with it. The sonification design will be discussed in Sec. 3.3.

A current field of research deals with the automatic analysis of power data in order to infer which appliances in a household are 
switched on and how much energy is used by them (so-called NonIntrusive Load Monitoring or NILM) [15]. However, NILM cannot be applied for data from standard smart meter devices without further information on the specifities of the plugged appliances. Alternatively, a wireless sensor network could be used to track the individual power of appliances. With the recent advancements towards low-energy sensors [16], this represents a viable alternative in terms of energy efficiency and yields the most precise results. In the long run, we aim at developing a system based on smart meter data. For developing our pilot system, we utilize individual measurement devices in order to comprehend our data better. The technical set-up of our pilot study consists of (1) five wall plugs of the intelligent home FIBARO system [17] sending data on power once a second over z-wave; (2) an AKG CK92 BlueLine omnidirectional microphone, an M-Audio MobilePre audio interface, and two Genelec 8020CPM loudspeakers; and (3) a laptop running PureData and SuperCollider for audio processing and DOMOTICZ [18], a software for smart home measuring devices receiving the data from the plugs.

\subsection{Electric power consumption in the kitchen}

Data of the kitchen appliances listed above have been recorded and analyzed to design the pilot system. The data provide rich patterns containing both information on power cycles typical of each appliance and on usage patterns typical of the kitchen users. The measured data are discontinuous due to the sampling of the measurement and the power draining behavior of the appliances. Thus, smoothing is needed and was implemented within the data preprocessing described in Sec. 3.2.

The power cycles of the appliances vary widely. Making coffee takes roughly one minute, during which the maker grinds coffee, boils the pre-heated water, and pours the coffee. The water kettle takes about two to three minutes depending on the amount of water being heated, and follows a step-function either being off or on. Furthermore, some appliances are directly connected to the users' interaction while others follow their own rhythm. Schwartz et al. [8] differentiate between an embodied service which stems from an activity personally performed by the user, while a background service is part of the domestic environment, e.g., the fridge with a regular cycle of cooling. The fridge's power cycle is hardly disturbed by user interventions, like, e.g., opening the door. The dish washer is an embodied service with a weekly, irregular cycle of usage. It is turned on two to three times a week, runs approximately for two hours, and drains most energy when it is hardly heard at all - i.e., when it is heating up water. Finally, the microwave is hardly used at all. All appliances furthermore drain power in standby, e.g., over night. One week of power of these different appliances is shown in Fig. 4(a).

The technical cycles of the appliances are overlaid by the pattern of the kitchen users. Fig. 4(b) shows the weekly average of three weeks' data. The presence of the institute's staff can be indirectly measured mainly by the coffee consumption showing a clear peak on Tuesday noon - where the institute's jour fixe takes place. Towards the weekend, the kitchen is less frequently used but the frequency of use is not reflected in the overall maximum of power. For instance, single runnings of the dishwasher dominate the overall power. We have considered ethical issues but concluded that sufficient anonymity is provided by not referring the data to our personal knowledge about the individual kitchen users' preferences.

\subsection{Data processing}

The two patterns of power described above are reflected in considerations on how efficient feedback can be given. On the one hand, successful feedback typically comprises appliance-specific data, offers interaction and presents information in an appealing and understandable way $[19,5,20]$. Feedback should be specific for a certain task and given in real time, e.g., continuously. Ambient ecological-feedback systems are promoted in the HCI community, and are mostly used for their motivational effects [8]. On the other hand, information has to be contextualized for the users, rather than displaying mere physical entities, as described by the same authors.

In this pilot study we explored in what ways the two levels of information can be conveyed using continuous auditory feedback. We decided to create a self-adapting system that both conveys direct feedback on the actual power and relates this information to the typical weekly user pattern. The algorithm of data preprocessing consists of an initialization and four iterative steps:

0 . Initializing baseline. The system is initialized with data of one average week of overall power, which has been gathered during the three weeks' monitoring period, see Fig. 4(b). This baseline contains $7 d \cdot 24 h \cdot 60 \mathrm{~m} \cdot 60 \mathrm{~s}=604800$ samples for each second of the week.

1. Smoothing the raw power. Most appliances exhibit discontinuous power patterns by nature of their on/off state, see Fig. 4(a). Therefore, the raw measurement data is smoothed with a leaky integrator that retains a fraction $a$ of the past output and adds $1-a$ of the actual input. (This is equivalent to low-pass filtering the data with a 1-pole IIR filter, or to exponential smoothing, see, e.g., [21].) Formulated for a series of digital samples, the filtered output at time $k, y(k)$, is calculated from the input at time $k, x(k)$, by

$$
y(k)=a \cdot y(k-1)+(1-a) \cdot x(k)
$$

where the smoothing constant $a$ relates the filter time constant $\tau$ and the sampling interval $T$ (here, $1 \mathrm{~s}$ ),

$$
a=e^{-\frac{T}{\tau}} .
$$

A suitable value for $\tau$ has been empirically adjusted in order to attain a good balance between sufficient smoothing and adequate temporal resolution for real-time feedback. In the first experiment, we have set $\tau=60 \mathrm{~min}$, which has lead to a clear weekly power pattern shown in Fig. 4(b) but to poor results for the real-time response in the first evaluation (Sec. 4.1). Therefore, we have reduced it to $\tau=15 \mathrm{~min}$. (After $5 \cdot \tau$, past data are decayed below $1 \%$ of their initial value.

2. Baseline update. The baseline is constantly being updated using another leaky integrator following Eq. 1 with $a=1 / 3$. This means that the actual smoothed power is iteratively averaged with the value of the past weeks with a weight of one third.

3. Power difference from baseline. For each second, the smoothed power is subtracted from the weekly baseline, resulting in the pattern shown in Fig. 4(c) for a randomly chosen week. The function is positive (resp. negative) if the smoothed (i.e., typical) consumption is above (resp. below) the baseline. 
4. Clipping outliers. The power difference is clipped along the $95 \%$ quantile of the baseline data in order to eliminate statistical outliers. The resulting value serves as driving signal for the sonification.

The steps 1., 2., and 3. are updated with new measurement data every second. Step 4 . is updated once a week, as no large variation of the $95 \%$ quantile of the baseline is expected.

\subsection{Sonification design}

Our pilot system is based on the mapping decision that a more reverberant room represents more power. Furthermore, the sound design is qualitatively different in two reverberation regions that are confined by three presets. The system has been implemented in SuperCollider, and in the final set-up we have used the plugin JPVerb [22]. The main differing settings of this algorithm for the presets are given in brackets below: $t 60$ is the reverberation time in seconds, i.e. the time the signal takes to drop by $60 \mathrm{~dB}$; damp is in the range of $[0,1]$ where 1 means strong damping for high frequencies; size varies between $[0.5,5]$ and gives the impression of a smaller/ larger space; finally, the reverberation time for the high frequency band has been slightly modified, with the parameter highcut for the crossover frequency, and the multiplying factor highx for the reverberation time of the high band:

Preset 0 - Zero: no augmentation/ no added reverberation (parameter settings as preset 1 but with amplitude $=0$.)

Preset 1 - Kitchen: additional reverberation which is so natural or plausible that only quick changes from or to this preset are perceived $(t 60=0.8$, damp $=0.0$, size $=1.1$, high $x=$ 0.3 , highcut $=1500$ )

Preset 2 - Church: the reverberation clearly exceeds plausible kitchen room acoustics, both in reverberation time and level $(t 60=3$, damp $=0.6$, size $=2$, highx $=0.5$, highcut $=1000$ )

We experimented with more artificial sounds, especially applying a pitch shift of up to $25 \mathrm{~Hz}$ on the incoming microphone signal. The resulting ringing echo was not well accepted in the first round of experiments and, therefore, dropped in the final version.

The driving signal of the sonification is mapped to reverberation in the way that preset 0,1 , and 2 coincide with lower threshold, zero-level and upper threshold in Fig. 4(c). In between, the levels of the parameters of the reverberation are interpolated linearly. Thus, the sonification is running and changing the room acoustics nearly all of the time (except for the 5\% statistical outliers of very low power difference, see Fig. 4(b)). In the lower region, where the smoothed actual power is lower than the baseline, the reverberation is changed very naturally. Only sudden and/or large changes in consumption will be perceived as change of room acoustics. In the upper region, however, the filtered actual power is above the baseline level and the reverberation quickly becomes unnatural. Our hypothesis was that the presets are tuned in a way that the change from just below to just above zero-level is obvious.

This sonification design allows to convey two different patterns. The first pattern is the change of the actual smoothed power. It is conveyed by a change in reverberation times and levels, and reacts immediately with the appliance itself (due to the smoothing it reaches the maximum at approximately $5 \tau$ in Eq. 1 or a shorter period if the initial value has been clipped). The second pattern is the level of the actual power compared to the baseline power. It allows to find out if the actual power is high or low as compared to the same weekday and time of the last weeks' average.

A demo video with binaural audio recording of the pilot system can be found on [23].

\section{EVALUATION OF THE PILOT STUDY}

The pilot study has aimed at assessing two factors, i.e. perceptibility and contextualization, and has been realized in two rounds of experiments. A first round of evaluation has centered on the question of perceptibility of the system. Furthermore, as the feedback is continuous and persistent, it needs to be accepted by the users. Following the paradigm of auditory augmentation, the auditory environment must not be changed in a way that the communication of the kitchen users is disturbed. Still, the feedback must not be too subtle in order to still being perceived. The second round of evaluation has questioned the contextualization of the system, specifically if and how well information on energy consumption is conveyed and if the energy awareness of the users is influenced.

The test subjects are our colleagues, i.e. the users of the institute's kitchen. These are 3 females and 12 males all of which have their workplace in the same building as the kitchen is. On the one hand, this group of subjects does not reflect a representative part of the population. On the other hand, they provide cumulated expertise as experienced listeners with a background in audio engineering, music, and/ or sound design.

\subsection{Evaluation part I}

The first round of experiment ran for ten days during the end of the the winter term 2017 , i.e. at a time when the kitchen is frequently used. The staff members had been informed beforehand that an experiment would be conducted in the kitchen involving the measurement of electrical power consumption. No further information had been given.

Following this first test period, a questionnaire was distributed to collect impressions of the system. Eleven subjects filled in the questionnaire. It contained standardized questions on kitchen use and open questions on the system. Results showed that the participants use the kitchen on a regular basis (7 people use the kitchen 3-5 times a week, 3 people use it 1-2 times, and only one less often). An overview of statements drawn from the questionnaire is shown in Fig. 2. The answers can be ordered along two continua: one is the level of perception/ control - from no audio-related perception to interactive control of the audio system. The other axis shows the range of negative and positive valence.

Asked whether they noticed anything during the test period in the kitchen only two participants denied. Thus, the majority of participants perceived the feedback. However, the results also show that the feedback was sometimes not perceived, perhaps occasionally due to the visiting time of the users. When the remaining nine participants were asked if they had noticed anything on all occasions, only three agreed, of which two were not sure about it. These findings show that the sonification reached its goal of staying at least partially below the threshold of perception (for cases when the actual power is below the baseline). The nine participants who have noticed something during the test period all correctly identified either resonant audio phenomena, feedback, or a changed soundscape. Without being asked explicitly, three subjects reported that they felt able to interact over speech or making 


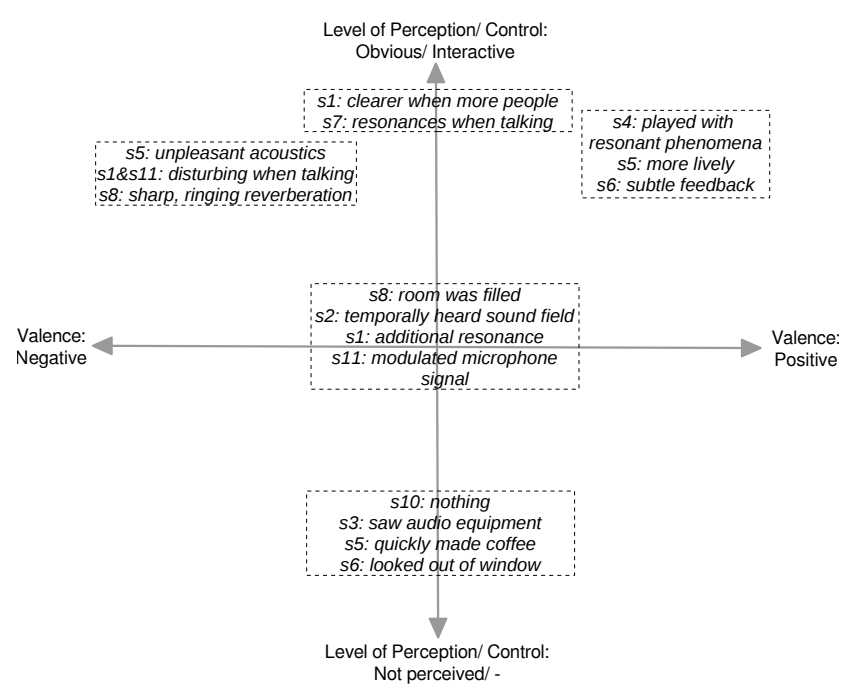

Figure 2: Qualitative statements by the subjects labeled s1 to s11 collected during the first experiment. The statements are ordered according to their level of perception/control of and valence towards the system.

other noises with the sound; one participant reported that his behavior had no influence.

The questionnaire contained only open questions. Most participants answered neutrally, but three complained about the sound. Two even turned the system off during a joint meeting (using the emergency switch of the loudspeaker). By contrast, two other participants gave positive answers about the 'subtle feedback'. They said that the kitchen was perceived more 'lively'. Three subjects asked specifically how the audio feedback is related to electricity consumption; none had worked out a hypothesis on the mapping.

The qualitative results above have led to modifications of the system, mainly the calibration of the parameters and the implementation of a better algorithm for reverberation. The smoothing constant of the actual power was reduced in order to give feedback more promptly. The preset 2 (Church) was tuned in order to reach a more pleasing sound quality even when the system is running at the maximum, i.e. at the upper threshold of the power difference.

\subsection{Evaluation part II}

The second round of evaluation aimed at assessing the user's possibility to contextualize the presented auditory information. Furthermore, the levels of control/perception and valence found in the first experiment have been explored more systematically, and completed with the dimension of arousal following research on affective reactions [24]. This evaluation has been conducted in the form of a diary study. The participants have been introduced to the system, both in terms of data preprocessing and the presets of the sound feedback. They have been requested to write a diary entry each time they would use the kitchen for a period of 2 two full weeks during the semester. The diaries have been miniquestionnaires asking for:

- Participant's name

- Date and time

- Duration and reason of stay in the kitchen
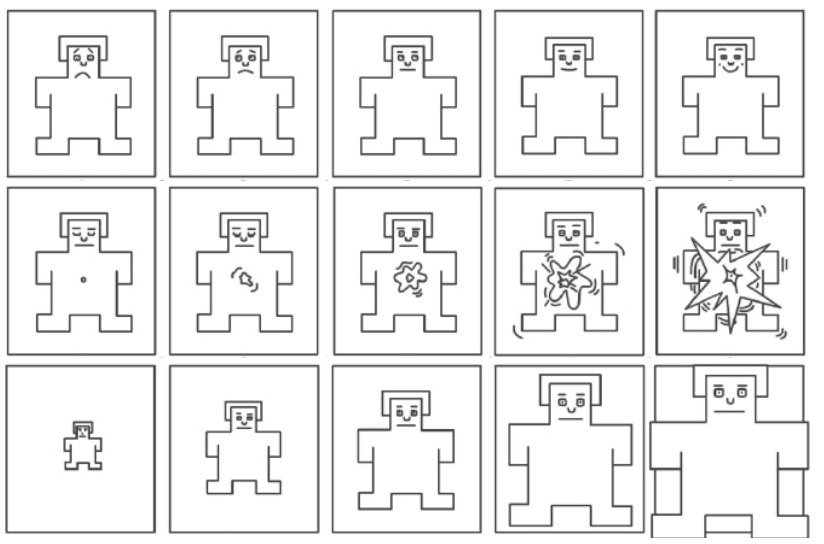

(a)

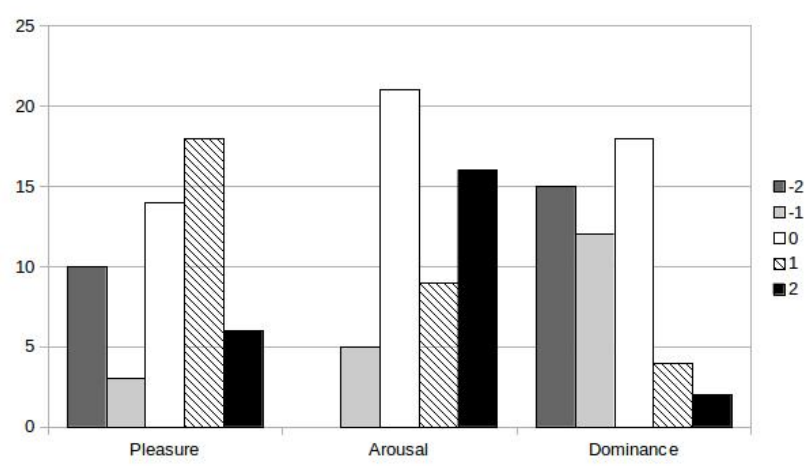

(b)

Figure 3: (a) 5-scale self-assessment manikin (SAM) for the dimensions valence, arousal, and control, measuring the affective reaction. The pictograms are taken from [25]. (b) For analysis the 5 states of the above diagram were given the numbers $-2,1,0,1$, and 2 , where 0 marks the neutral manikin in the middle. The results for the SAM rating show a wide spread of affective responses in the diary entries.

- Whether the soundscape has attracted any attention:

- A description of the situation

- A 5-scale self-assessment manikin (SAM) for the dimensions control, valence, and arousal, measuring the affective reaction of a users towards the sound system, see Fig. 3(a). The SAM technique has been established for quick and non-verbal testing and has been successfully used to evaluate sound, e.g., [24].

- Any other reactions.

The collected diary entries have been analyzed both quantitatively and qualitatively. In total, 14 users took part in the survey. Six users filled in their diary entries on a regular basis, with 13,6 entries per person. Eight more users only handed in 1-3 entries each. Not all of them could be taken into account, because the system has occasionally been turned off by the users and was only re-started with some delay. The ratio between entries where people reported perceiving something and not perceiving anything was about $50 \%$. Astonishingly, these data show no correlation with the driving value of the sonification. The hypothesis, that the sonifi- 
cation is clearly detected above the threshold of 0 is not supported by the data. This could be due to statistical reasons, with a sample size of approximately 60 entries that could be taken into account for the statistical analysis. Another explanation might be deduced from the qualitative answers. The diary entries showed large interpersonal differences. While some people appear to be very sensitive towards the mapping, describing the reverberation as "strong" or "reverberation of a church" even for values just above 0 , others have not remarked anything until much higher values. As a conclusion on these results, the calibration of the mapping has to be thouroughly tested with the participants, and the participants have to become acquainted with the mapping in a longer learning phase.

Qualitative analysis of the SAM rating supports the findings above. Different kitchen users had very different affective reactions towards the system. Some being positive in general, some negative, and some varied their rating from entry to entry. The bar chart in Fig. 3(b) gives an overview of the heterogeneous ratings. For the dimension of pleasure the mean value is not significantly different to 0 (mean $=0.13$ with confidence interval of $+/-0.36$, calculated for a significance level of $\alpha=5 \%$ and $n=60$ samples), i.e., positive and negative attitudes towards the system are in balance. Arousal has a mean of $0.70+/-0.28$, thus the system has an arousing factor. In the case of dominance, the mean value is $-0.66+/-0.31$, thus subjects feel to be slightly passive towards the system.

\section{CONCLUSION AND OUTLOOK}

This paper describes a system of auditory augmentation that conveys information on electric power consumption of an institute's kitchen. Data collected from five kitchen appliances contain two patterns: one stemming from the technical cycle of each appliance, and a second one from the interaction of the kitchen users. The data have been pre-processed in order to give information on both levels. The smoothed actual power consumption relative to a baseline controls the level of reverberation within two sound presets: a 'typical' kitchen use leads to a typical kitchen reverberation, while a particularly high power consumption leads to the reverberation of a church.

The results of the evaluation indicate that the state of the system was not perceived saliently. One problem of the approach of auditory augmentation in the kitchen is that the stationary noise of running appliances interferes with the perceptability of the reverberation. For instance, the water kettle or dishwasher sounds are broadband noise signals that mask the perception of reverberation. By contrast, reverberation is much better perceivable if the source sound is short and transient. These kinds of sounds stem from the interaction of the users, e.g., of footsteps or when placing a cup on the countertop. Thus, the ambient auditory display is more perceptible when excited by users' sounds than by the sounds of appliances. One solution could be to adjust the reverberation level dynamically to the level of the soundscape.

In general, sustainable HCI systems which aim at encouraging users to save energy face the conflict that they themselves utilize resources. For a prototype design, this can be accepted. In an optimized product, the net energy balance has to be weighed.

We consider a few design changes for a next, possibly in-home experiment. It might be useful to separate embodied services from background services. In our system, this would mean using a different smoothing constant for the fridge. Its regular cycles could be smoothed out, as feedback on whether the fridge drains power or not is not relevant to the users.

Further evaluation is envisaged, e.g., conducting an experiment in the kitchen with participants placed in the room for a few hours, giving them a task, and checking if feedback on the power is still being perceived at the background of their attention. In a next step, the system should be tested in a real in-home setting.

The presented system is deliberately simple and neutral, i.e. completely data-driven and not judgmental with respect to an absolute level of consumption. On the contrary, it is self-adaptive, constantly updating the weekly baseline. A shortcoming of the system is its real-time nature, giving feedback about now and how this time and date relates to the respective previous ones. Times in which the kitchen are not or hardly used, e.g., at night, are not taken into account. Turning appliances off during the night is possibly the largest factor for saving energy at our institute's kitchen. In a future version of the installation this fact should be included in the sonification design.

\section{ACKNOWLEDGEMENT}

This research was supported by the Cluster of Excellence Cognitive Interaction Technology 'CITEC' (EXC 277) at Bielefeld University, which is funded by the German Research Foundation (DFG).

\section{REFERENCES}

[1] European Environment Agency, "Final energy consumption of electricity by sector." [Online]. Available: http://www.eea.europa.eu/data-and-maps/indicators/ final-energy-consumption-by-sector-9/assessment-1

[2] European Commission, "Benchmarking smart metering deployment in the eu-27 with a focus on electricity," REPORT FROM THE COMMISSION, 2014.

[3] European Commission, Directorate-General of Communications Networks, Content \& Technology, "Definition of a research and innovation policy leveraging cloud computing and iot combination, final report." [Online]. Available: http: //ec.europa.eu/newsroom/dae/document.cfm?doc_id=9472

[4] R. Hierzinger, M. Albu, H. van Elburg, A. Scott, A. Łazicki, L. Penttinen, F. Puente, and H. Sæle, "European smart metering landscape report, smartregions deliverable 2.1," vol. 24, p. 2013, 2012.

[5] S. Darby, "The effectiveness of feedback on energy consumption: a review for DEFRA of the literature on metering, billing and direct displays." Environmental Change Institute, University of Oxford, Tech. Rep., 2006. [Online]. Available: http://www.eci.ox.ac.uk/research/ energy/electric-metering.php

[6] Y. Ishihara, M. Ishihara, F. Hirayama, and K. Yasukawa, Domestic Electricity Consumption Visualized as Flowing Tap Water to Raise the Feeling of Waste. Cham: Springer International Publishing, 2015, pp. 546-550. [Online]. Available: http://dx.doi.org/10.1007/978-3-319-21383-5_91

[7] A. Harman, H. Dimitrov, R. Ma, S. Whitehouse, Y. Li, P. Worgan, T. Omirou, and A. Roudaut, "Notifall: Ambient sonification system using water," in Proceedings of the 2016 CHI Conference Extended Abstracts on Human Factors in Computing Systems, ser. CHI EA 
'16. ACM, 2016, pp. 2667-2672. [Online]. Available: http://doi.acm.org/10.1145/2851581.2892443

[8] T. Schwartz, G. Stevens, L. Ramirez, and V. Wulf, "Uncovering practices of making energy consumption accountable: A phenomenological inquiry," ACM Trans. Comput.-Hum. Interact., vol. 20, no. 2, pp. 12:1-12:30, May 2013. [Online]. Available: http://doi.acm.org/10.1145/ 2463579.2463583

[9] T. Bovermann, R. Tünnermann, and T. Hermann, "Auditory Augmentation," International Journal on Ambient Computing and Intelligence (IJACI), vol. 2, no. 2, pp. 27-41, 2010.

[10] R. Tünnermann, J. Hammerschmidt, and T. Hermann, "Blended sonification -sonification for casual information interaction," in Proceedings of the International Conference on Auditory Display (ICAD), 72013.

[11] F. Kilander and P. Lönnqvist, "A whisper in the woods - an ambient soundscape for peripheral awareness of remote processes," Proceedings of the International Conference on Auditory Display (ICAD), 2002.

[12] E. Brazil and M. Fernström, "Investigating ambient auditory information systems," Proc. of the International Conference on Auditory Display (ICAD), 2007.

[13] A. Parthy, C. Jin, and A. van Schaik, "Reverberation for ambient data communication," Proceedings of the International Conference on Auditory Display (ICAD), 2004.

[14] W. Ritsch, T. Musil, A. Sontacchi, J. Zmölnig, and F. Zotter, "The cubemixer a performance-, mixing- and masteringtool," in Proceedings of the LAC 2008 (Linux Audio Conference 2008), F. Barknecht and M. Rumori, Eds.

[15] M. Zeifman and K. Roth, "Nonintrusive appliance load monitoring: Review and outlook," IEEE Transactions on Consumer Electronics, vol. 57, no. 1, pp. 76-84, 22011.

[16] J. Rabaey, J. Ammer, J. L. da Silva, and D. Patel, "Picoradio: Ad-hoc wireless networking of ubiquitous low-energy sensor/monitor nodes," in Proceedings IEEE Computer Society Workshop on VLSI 2000. System Design for a Systemon-Chip Era, 2000, pp. 9-12.

[17] Fibaro, "Wall plug," 2017. [Online]. Available: http: //www.fibaro.com/uk/the-fibaro-system/wall-plug

[18] Domoticz. (2017) Home automation system. [Online]. Available: https://domoticz.com/

[19] W. Abrahamse, L. Steg, C. Vlek, and T. Rothengatter, "A review of intervention studies aimed at household energy conservation," Journal of Environmental Psychology, vol. 25, no. 3, pp. 273 - 291, 2005. [Online]. Available: http://www.sciencedirect.com/science/article/pii/ S027249440500054X

[20] C. Fischer, "Influencing electricity consumption via consumer feedback. a review of experience," in European Council for an Energy Efficient Economy (ECEEE) (ed.):Energy Efficiency: Just do it!ECEEE 2007 Summer Study Proceedings, 2007, pp. 1873-1884.

[21] Performity LLC, "Exponential filter," 2018. [Online]. Available: http://gregstanleyandassociates.com/ whitepapers/FaultDiagnosis/Filtering/Exponential-Filter/ exponential-filter.htm
[22] GitHub, "Extension plugins for the supercollider3 audio synthesis server." [Online]. Available: https://github.com/ supercollider/sc3-plugins

[23] Anonymized, "Supplementary material (link to be exchanged by data-doi for camera-ready version)," 22018. [Online]. Available: https://goo.gl/NiWs7j

[24] F. Stevens, D. Murphy, and S. Smith, "Emotion and soundscape preference rating: using semantic differential pairs and the self-assessment manikin," in Sound and Music Computing conference, Hamburg, vol. 2016, 2016.

[25] A. P. Soares, A. P. Pinheiro, A. Costa, C. S. Frade, M. Comesaña, and R. Pureza, "Affective auditory stimuli: Adaptation of the international affective digitized sounds (iads-2) for european portuguese," Behavior Research Methods, vol. 45, no. 4, pp. 1168-1181, Dec 2013. [Online]. Available: https://doi.org/10.3758/s13428-012-0310-1 


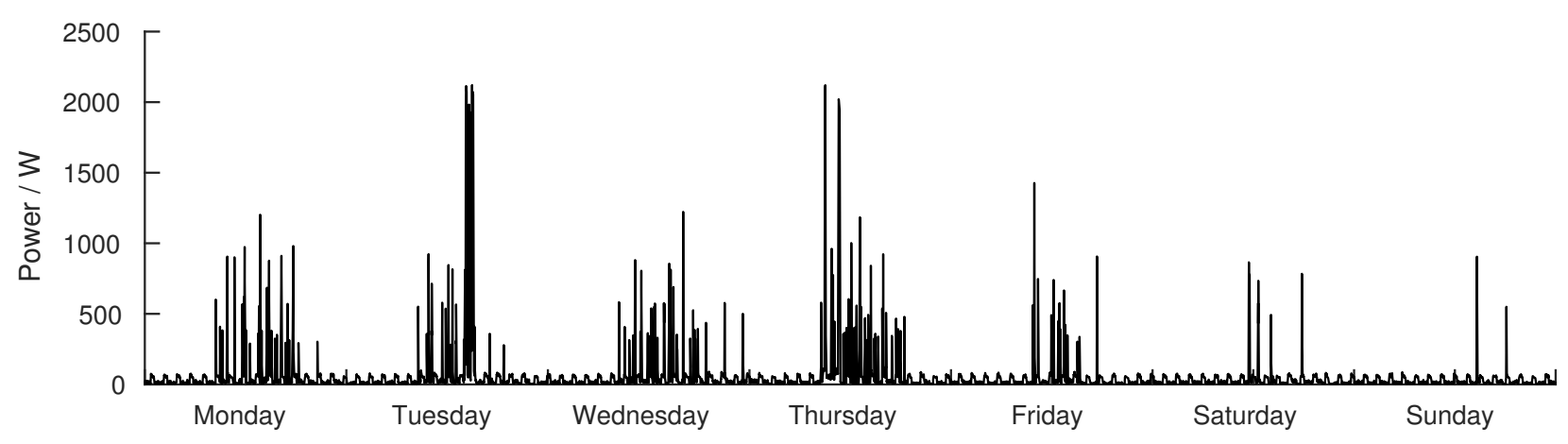

(a)

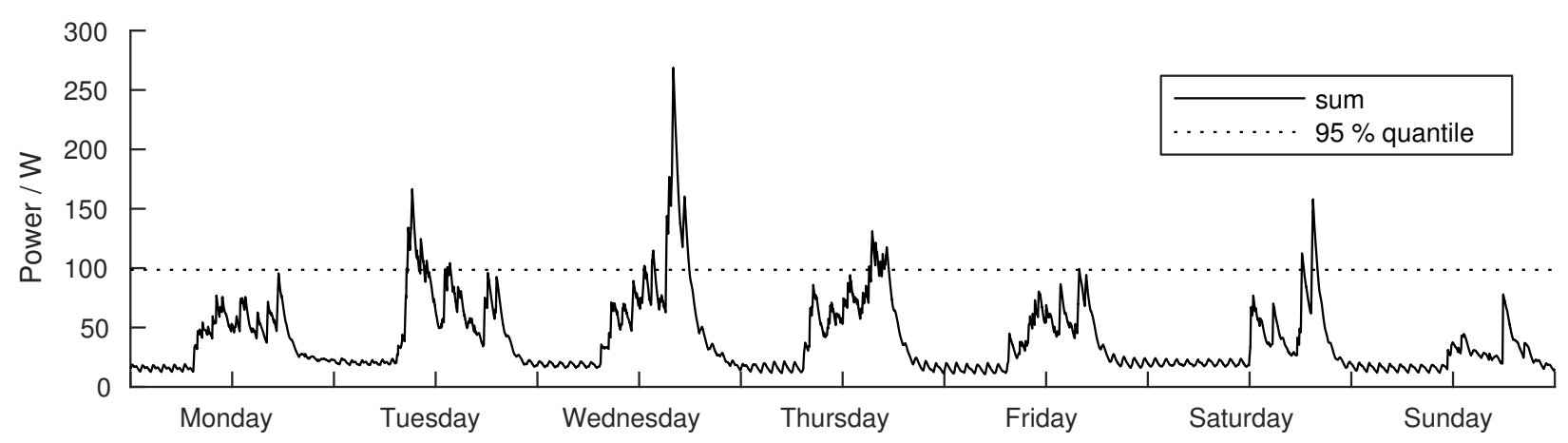

(b)

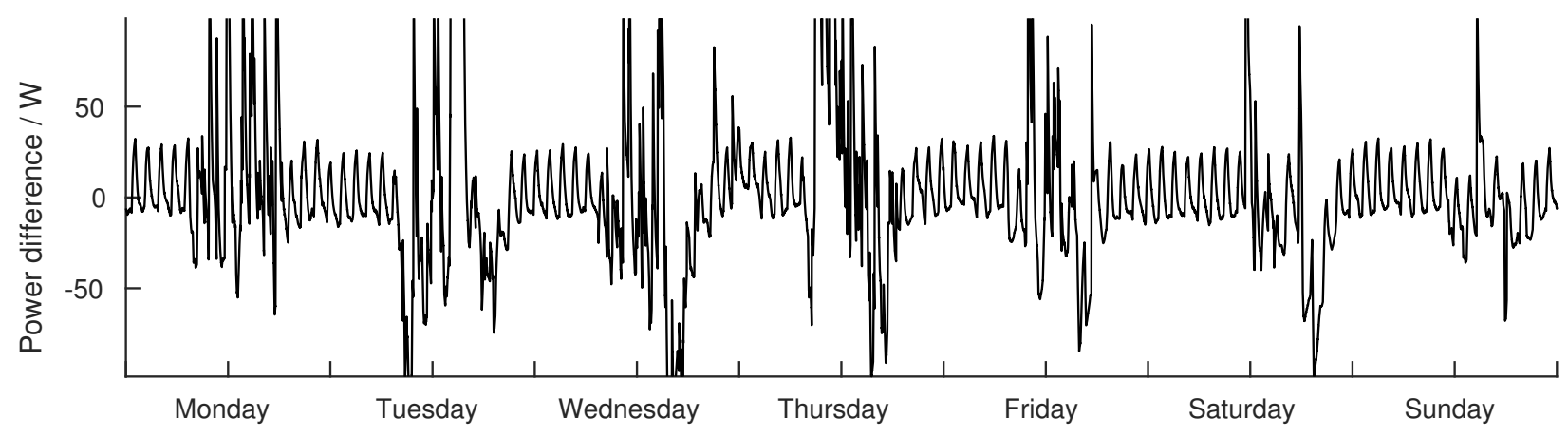

(c)

Figure 4: (a) One week of electric power consumption during the second evaluation study, 12-18 March 2018. The data have been smoothed for this plot with $\tau=1 \mathrm{~min}$ to show the actual, short-time power consumption. The two largest peaks are due to runnings of the dishwasher on Tuesday and Wednesday of that week. During weekdays there are many smaller peaks due to coffemaker and the water kettle. Finally, the small periodicity that can be observed during the night is due to the cooling rhythm of the fridge. It continues throughout the week but is masked (in this plot) by higher consumption during the day. (b) Average weekly power derived from three weeks' data of a different period, in Autumn 2017. The dotted line shows the $95 \%$ quantile that separates 5\% of outliers of peak current. The filter time constant $\tau$ for generating these plots is one hour. (c) Power difference of the actual power shown in (a), smoothed with $\tau=15$ min, minus the weekly baseline shown in (b). The data are clipped at the lower and upper thresholds that correspond to the $95 \%$ quantile of the baseline data. This clipped power difference is the driving signal for our sonification approach. 